\title{
Disclination-mediated thermo-optical response in nematic glass sheets
}

\author{
Carl D. Modes, ${ }^{1}$ Kaushik Bhattacharya, ${ }^{2}$ and Mark Warner ${ }^{1}$ \\ ${ }^{1}$ Cavendish Laboratory, University of Cambridge, Madingley Road, Cambridge CB3 OHE, United Kingdom \\ ${ }^{2}$ Division of Engineering and Applied Science, California Institute of Technology, Pasadena, California 91125, USA
}

(Received 26 October 2009; revised manuscript received 3 May 2010; published 8 June 2010)

\begin{abstract}
Nematic solids respond strongly to changes in ambient heat or light, significantly differently parallel and perpendicular to the director. This phenomenon is well characterized for uniform director fields but not for defect textures. We analyze the elastic ground states of a nematic glass in the membrane approximation as a function of temperature for some disclination defects with an eye toward reversibly inducing three-dimensional shapes from flat sheets of material, at the nanoscale all the way to macroscopic objects, including nondevelopable surfaces. The latter offers a paradigm to actuation via switchable stretch in thin systems.
\end{abstract}

DOI: 10.1103/PhysRevE.81.060701

PACS number(s): 61.30.Jf, 46.32.+x, 46.70.De, 46.70.Hg

Nematic glasses (densely cross-linked networks) [1] are solids with a natural state of elongation along their director and contraction perpendicular that depends on their orientational order. Accordingly they suffer large, reversible length change with heating [2], illumination [3,4], solvent uptake, $p \mathrm{H}$ change and any other stimulus that causes order change. For glasses these strains can be $2-3 \%$ and of opposite sign (without conserving volume) along and perpendicular to the director. They can then exhibit spectacular effects such as large bend resulting from gradients of stimuli [5] (light, solvent) or from uniform stimuli (such as temperature or weakly absorbing light) but with a director gradient through, for instance, the section of a sheet or cantilever [2-4] (usually twist or splay-bend). Glasses are distinguished from elastomers by being so heavily cross-linked that the director field only changes as a result of convection due to material shape change from the elastic strain. Nematic glass directors do not rotate relative to the matrix as in nematic elastomers-they are [3] conventional uniaxial elastic solids, with moduli of about $\times 10^{4}$ higher than those of elastomers. We are most concerned with the thermally or optically inspired length changes which we take to be by a factor $\lambda$ along the director and by $\lambda^{-\nu}$ perpendicular to the director (where $\nu$ fulfills the role of a thermal or optical Poisson ratio, were the deformations to be infinitesimal). Elastic energy cost would be associated with imposed changes away from these new natural shapes-we find deformation fields matching these thermo/ optical changes and thus of zero elastic energy in the membrane limit we take. Accordingly we do not need to explore the solid body elasticity specific to uniaxial materials.

Director fields can be established in the nematic liquid progenitor phase before cross-linking and are permanently recorded in the solid state achieved after linkage. In fact complex, three-dimensional (3D) director fields for subtle mechanical response can be achieved in nematic glasses [6] via holography and surface preparation. From the point of view of device design, the ability to "write" an initial director field into a solid so that it distorts in a predictable (and reversible way) into a new shape with applied temperature or light would be ground breaking. In particular, the ability to take flat thin sheets of material and turn them into prescribed potentially complicated nondevelopable shapes-at nearly any length scale-is highly sought after. Accordingly, we consider here the elastic response of thin sheets of nematic glass in the membrane approximation. We are interested in thin sheets where the director is uniform through the thickness. In this situation, a change in nematic order gives rise to in-plane stretches (or contractions). If the initial director field is not homogeneous, then this change in nematic order gives rise to inhomogeneous changes of stretch that may or may not be compatible. A key observation here is that thin sheets can accommodate many more potential inhomogeneous changes in stretch compared to bulk 3D specimens by possibly deforming out of plane-in our case cones and anticones with localized Gaussian curvature, see Fig. 1. Further, in thin sheets, the energy and forces associated with membrane mode (in-plane stretch) scales as the thickness while those associated with bending mode (differential in-plane stretch) scales as the third power of thickness. So the bending energy is negligible compared to the in-plane stretches. All of this leads to a new paradigm for actuation [7]: if we are able to find a director arrangement that leads to stretches that are incompatible in bulk but compatible in sheets, then the change in order can be used to generate out-of-plane deformations with a very large blocking force (one that scales with thickness). This is in contrast with small blocking forces (third power of thickness) associated with designs involving bending cantilevers. We show that +1 disclinations are indeed such arrangements.

Disclination defect textures are not extensively studied, experimentally or theoretically, in nematic solids. However it is known that the energy minimizing deformation associated locally with changes in their nematic order is not compatible in some $3 \mathrm{D}$ disclinations [8]. We analyze the mechanical

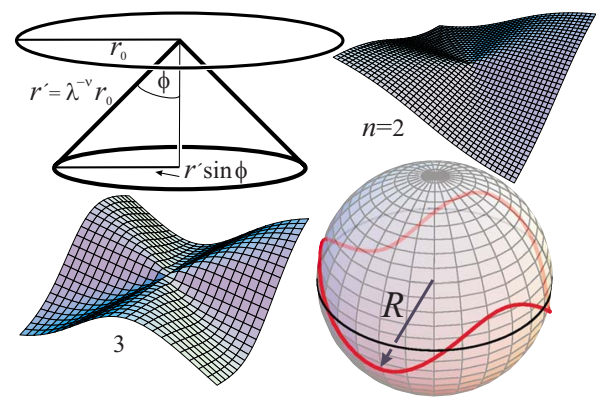

FIG. 1. (Color online) A flat nematic glass sheet with an azimuthal +1 disclination heats to a cone or cools to "anticones" $(n)$. 


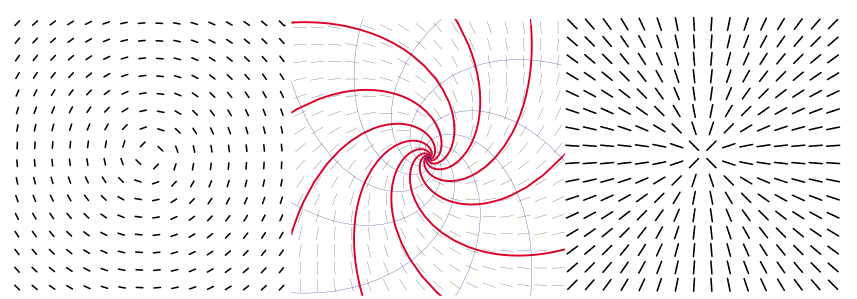

FIG. 2. (Color online) +1 disclinations-azimuthal and radial textures have as intermediates logarithmic spirals (shown with $\delta$ $=45^{\circ}$ ). The heavy lines (red, online) are the integral curves of the director field, and those of its complement are light (blue).

response and elastic ground state of the most experimentally accessible disclination defect in two dimensions that with topological charge +1 . We argue that it is a powerful way to induce shape change, specifically through the introduction of a point of localized Gaussian curvature. Figure 1 shows examples of initially flat defected sheets after heating or cooling.

We illustrate some ways director fields with this disclination charge could be used in concert with system geometry for applications, such as sharp bending, twisting, or forming nondevelopable (anticlastic) surfaces and cantilevers.

Shape response to nematic defects has been investigated theoretically in fluid systems $[9,10]$ where the dominant influences include surface tension, Frank elasticity, and ordercurvature coupling. Our solids are in the opposite limitelastic stresses dominate over Frank elasticity for length scales greater than a nematic penetration depth for solids $\xi$ $=\sqrt{K / \mu}$, where $\mu$ and $K$ are the shear modulus and Frank constant, respectively. ( $\xi \sim 10^{-10} \mathrm{~m}$ for a glass.) Also, in our problem surface energies, $\sigma$, play no role; competition with solid body elasticity produces a length scale $\sigma / \mu$ of comparable smallness to $\xi$ and such effects are also irrelevant.

Response closely analogous to ours has been analyzed by Ben Amar et al. in the elasticity of botanical systems when anisotropic growth creates internal stresses and forces planar systems into the third dimension $[11,12]$ as cones or "econes" (our anticones). They and we deal with localized Gaussian curvature and hence localized elastic stretch. Such stress intensification occurs in folding and crumpling, see Witten's review [13]. However, our cones and anticones are simple, not "d-cones" [13], and our systems naturally take up such shapes rather than concentrate stress in response to spatial crowding. Indeed, except for very weak spontaneous distortions, our tip extent is of the order of the thickness as in classical simple cones [13]—we return elsewhere to the core or far-field bend or stretch energy balance. Thus our system differs from the cone sources that generate crumpling.

We consider +1 disclinations. In the membrane limit in which we choose to work, escape in to the third dimension [14] of the director field is not possible except perhaps near the core, and these disclinations are true topological defects. For +1 defects, many different textures, though topologically equivalent (see Fig. 2), differ nontrivially in their mechanical response. We first analyze azimuthal and radial textures. $m$ $\neq 1$ defects present considerable elastic compatibility problems, even in the 2D forms of this Rapid Communication, and we consider their mechanical effects elsewhere.
Consider a thin sheet of nematic glass whose director field is azimuthal around $\mathrm{a}+1$ disclination defect, as in the left of Fig. 2, and which is flat at some reference temperature, $T_{0}$. As the sample is heated above $T_{0}$, the decline in nematic order will cause a contraction of the natural length along the nematic directors with a local elongation of the natural length due to Poisson effects normal to them. In a free uniform glass these natural length changes would be manifested by actual mechanical strains so that the elastic ground state is achieved. Since the chosen director field is circularly symmetric, with integral curves simply concentric circles centered on the defect, clearly the sheet has a problem accommodating this change in the natural lengths as circumferences shrink while the corresponding natural radii grow.

Fortunately, in the membrane approximation where we may neglect bending energies, there is an obvious geometric solution that allows the nematic glass to respond to the imposed thermal strain without paying the high energetic cost associated with elastic compressions and expansions relative to this changed state-a cone. This may be seen intuitively by keeping track of the deformation of a circle of material centered on the defect. At $T_{0}$ the sheet of nematic glass is flat, and the circle maintains the familiar perimeter-to-radius ratio of $2 \pi$. However, as the temperature rises, the perimeter wants to change by a factor of the thermal deformation gradient along the director, $P \rightarrow P^{\prime}=\lambda P$, where here $\lambda<1$. Meanwhile, see Fig. 1, the material (in-plane) radius is changing as well due to the thermal or optical Poisson effects associated with the perimeter's change, $r_{0} \rightarrow r^{\prime}=\lambda^{-\nu} r_{0}$. For glasses $\nu$ is in the range $1 / 3$ to 2 [3]. Together, these transformations imply that upon heating, a circle of material on the sheet remains circular but adopts a new in-material perimeter-to-radius ratio that of a circle enclosing a cone's tip (Fig. 1, first panel):

$$
\begin{aligned}
P^{\prime} & =2 \pi \lambda^{1+\nu} r^{\prime} \rightarrow 2 \pi r^{\prime} \sin \phi \\
& \rightarrow \phi\left(T-T_{0}\right)=\sin ^{-1}\left(\lambda^{1+\nu}\right),
\end{aligned}
$$

with $\phi$ as the cone opening angle. One can think of $r^{\prime} \sin \phi$ as the embedded radius. The localized Gaussian curvature associated with the cone tip is thus $2 \pi(1-\sin \phi)$; circles enclosing the tip of a nondevelopable cone no longer have the ratio $2 \pi$ of the perimeter to the in-plane radius, unlike circles on the cone but not enclosing the tip. Away from the singularity, there is no Gaussian curvature and hence no shapeinduced elastic compressions or extensions. Therefore our sheet of nematic glass responds to temperatures above $T_{0}$ by deforming out of plane, breaking up-down symmetry in the process. The opening angle varies sensitively with small strains; a $2-3 \%$ contraction with $\nu \sim 2$ achieved over $90{ }^{\circ} \mathrm{C}$ [2] gives $\phi \sim 70^{\circ}-66^{\circ}$, such strains also being achievable for modest illuminations in photoglasses [4].

But what if we cool the azimuthal sample below $T_{0}$, increasing the nematic order relative to the reference state? The arguments relating the local changes in length along and normal to the nematic director go through unchanged, however now $\lambda>1$, invalidating our previous ansatz cone 
solution-now the perimeter is "too long" for the in-plane radius and a more complex deformation must result.

We consider deformed surfaces where the height varies linearly with the distance from the center or the defect. Consider a circle of radius $r_{0}$ centered at the defect in the flat undeformed reference plane. After deformation, this circle becomes a curve described by $\{r(\phi), \phi, h(\phi)\}$ in cylindrical coordinates $(r, \phi, h)$ in the deformed configuration and parametrized by the azimuthal angle $\phi$. If the material is in the minimal energy state unstretched from its new natural configuration, this curve has to satisfy two constraints. First, it has to have a constant distance $R=\lambda^{-v} r_{0}$ from the origin (defect) and second, its length has to be equal to $P=2 \pi \lambda r_{0}$ $=2 \pi \lambda{ }^{1+\nu} R$. Thus, the curve has to lie on a sphere of radius $R$; see the trajectory in the last panel of Fig. 1. Further, since the length of the curve $P$ is greater than the length $2 \pi R$ of the great circle of the sphere, this curve has to oscillate. In other words, the deformed surfaces oscillate azimuthally and these oscillations grow linearly radially as shown in Fig. 1. We call them anticones. We also note

$$
\begin{gathered}
R=r \sqrt{1+\left(\frac{h}{r}\right)^{2}}, \\
P=\int_{0}^{2 \pi} d \phi \sqrt{\left(\frac{d r}{d \phi}\right)^{2}+\left(\frac{d h}{d \phi}\right)^{2}+r^{2}} .
\end{gathered}
$$

The simplest possibilities for $h$ are

$$
h(r ; A, n)=A r \sin n \phi
$$

for antinodal line angle $\alpha=\tan ^{-1} A$ (amplitude in effect; see Fig. 1) and integer $n$ (so that the curve is closed). Plugging this ansatz into Eq. (2) gives the relationship between the $r$ coordinate and $\phi$ at constant $R$ needed for the perimeter:

$$
r=\left(1+A^{2} \sin ^{2} n \phi\right)^{-1 / 2} R .
$$

Returning this relation and the form of $h$ to Eq. (3) for the perimeter gives grouping factors of $R$ and simplifying

$$
P(R)=2 \pi R I(n, A),
$$

where $I(n, A)$ depends only on the scale $A$ and the state $n$ :

$$
I(n, A)=\int_{0}^{1} d u \sqrt{\frac{n^{2} A^{2} \cos ^{2} 2 \pi n u}{\left(1+A^{2} \sin ^{2} 2 \pi n u\right)^{2}}+\frac{1}{1+A^{2} \sin ^{2} 2 \pi n u} .}
$$

Connecting the radius and perimeter as in Eq. (1) gives here $I(n, A)=\lambda^{1+\nu}\left(T-T_{0}\right)$; as temperature and hence spontaneous distortion changes, so does the character (that is, $A$ and $n$ ) of the anticone. The negative Gaussian curvature localized at the apex of the anticone is $2 \pi(1-I)$. As appropriate, $I=1$ for $n=0$ - the surface is a flat plane. Otherwise $I$ ranges from 1 to $|n|$ for $A=0 \rightarrow \infty$, see Fig. 3 .

The analogous cosine solutions simply give rise to rotated versions of the same surfaces for all $n \neq 0$, recovering the conical solution discussed earlier for $n=0$. In this case $I(A ; n=0)$ ranges from 1 at $A=0$ to 0 at $A=\infty$, as required. The behavior of $I$ for $n \neq 0$ is encouraging-our trial solutions yield precisely the geometries that accommodate at zero stretch energy a cooling of our azimuthal +1 defect

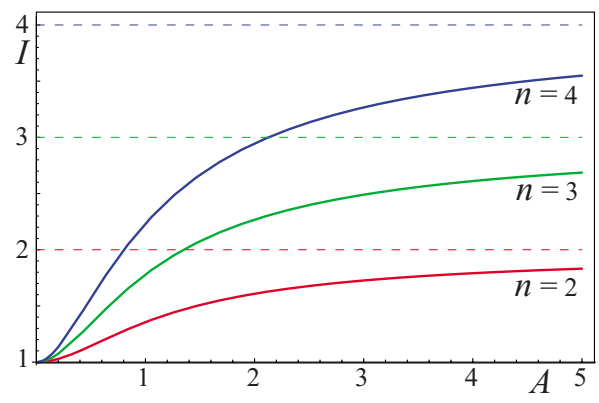

FIG. 3. (Color online) The behavior of $I(n, A)$ for different values of $n$ and as a function of $A$. When $A$ is large, $I(n, A)$ approaches $n$.

below $T_{0}$. However, since each individual surface is limited to a maximum perimeter-to-radius ratio of $2 \pi|n|$, we would expect interesting transition behavior as cooling leads to strains requiring ever more crumpled geometries, with transition states characterized by simple Fourier combinations of the "pure" surfaces or, in extreme cases, exotic surfaces that are multiply re-entrant in $\phi$. Figure 1 shows an $n=3$ anticone where the crumples take up more perimeter than the more slowly varying $n=2$ anticone. See [12] for an analysis of large amplitude anticones where the functions $h(\phi), r(\phi)$ may not be single valued. We conjecture that all minimal membrane energy solutions of these defects are anticones.

A paradigm for actuation now arises. Although we are dealing with thin sheets (and later cantilevers) in the membrane approximation where shape change has low bending energy, large forces can be exerted by switching on and off Gaussian curvature via stretch modes that arise if the natural and imposed geometries are in conflict.

For radial textures (Fig. 2), the roles of the direct thermal strain and Poisson strain are swapped. Heating above $T_{0}$ now requires a shortening of the radial length due to decreased nematic order while the azimuthal direction expands from the corresponding Poisson effect-equivalent to lowering the temperature in the azimuthal texture. Hence, each of these textures behaves as the other under the mapping $T-T_{0}$ $\rightarrow T_{0}-T$.

Generically $\mathrm{a}+1$ disclination defect has an intermediate angle $\delta$ of the director with respect to the radial vector from the defect core. As a result, the effect of the direct and Poisson strains are now mixed along circles and radii centered on the defect. Curves along which the material feels the maximal effect of the direct strain and none of the Poisson strain and vice versa are the integral curves of the director field and its normal complement, respectively. For +1 textures with $0<\delta<\pi / 2$, the integral curves are logarithmic spirals instead of simple circles and radii (Fig. 2).

An analysis we present elsewhere shows that the spiral angle $\delta$ evolves with spontaneous deformation $\lambda$. Defining $b=\cot \delta$, one finds that $b \rightarrow b / \lambda^{1+\nu}$. For instance for heating where $\lambda<1$, then the angle $\delta$ decreases-the spiral tends more to the radial direction. Also a mismatch between radius and perimeter as considered above arises: cones or anticones form depending on whether the radius or perimeter grows relative to the other. For instance for an initial $\delta>\pi / 4$ and hence $b$ initially $<1$, the spiral gives a conical response for 
$\lambda \in\left(b^{2 /(1+\nu)}, 1\right)$ and anticones otherwise. The cone angle is $\phi=\sin ^{-1}\left[\frac{\lambda^{2(1+\nu)}+b^{2}}{1+b^{2}} \frac{1}{\lambda^{1+\nu}}\right]$.

There is a new effect, however, because of the spiral angle of the director relative to the radial direction, material undergoes motion with an azimuthal component. Furthermore, if $\delta$ and $\lambda$ are such that an out-of-plane deformation into a cone is required, then this rotation, combined with the material's spontaneous choice to form an "upward" or "downward" pointing cone as the material moves from two to three dimensions, leads to a spontaneously broken chiral symmetry. This symmetry breaking does not occur for the anticone solutions, as they do not break up-down symmetry.

Consider advantageous ways to use such sheets other than as cone or anticone machines. Simplest would be to break the circular symmetry by cutting out a cantilever of material. Strips cut from these defected textures can display a wide range of rich behavior (Fig. 4): cusped with sharp bending, pure twist, curvature reversal, and combinations of these depending on the orientation of the cantilever with the defect. Blocking forces would depend on the dimensions of the (anti)conical region compared with the length of the arms. Boundary conditions are very important, and the cut out material must encompass the defect core. Such active response in strips could be used as a light activated stirrer, actuator, swimmer, or perhaps as thermally sensitive simple machines.

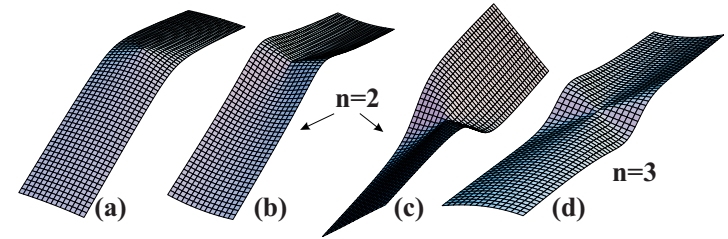

FIG. 4. (Color online) Disclinations in cantilevers also cause unusual thermal or optical response. (a) a strip cut from a +1 texture conically deforming (with $\phi=45^{\circ}$ ) bends with a conical cusp. Anticonical deformations are even richer (here $\alpha=45^{\circ}$ ), with (b) anticlastic bending ( $n=2$, antinodes aligned with the cantilever axes) (c) pure twist (nodes aligned) or (d) curvature reversal $(n=3)$.

"Frozen-in" disclinated director fields in responsive nematic glasses are rich and promising systems. To exploit them to their fullest however, will rely on understanding the mechanical response of all the defect charges, along with how interacting multiple defects influence the resultant strainmediated shape change. Such understanding would allow blueprinting an arbitrary three-dimensional shape in a flat sheet and switching it on at will.

C.D.M., K.B., and M.W. acknowledge support from the EPSRC-GB.
[1] D. J. Broer and G. N. Mol, Polym. Eng. Sci. 31, 625 (1991).

[2] G. N. Mol, K. D. Harris, C. W. M. Bastiaansen, and D. J. Broer, Adv. Funct. Mater. 15, 1155 (2005).

[3] K. D. Harris, R. Cuypers, P. Scheibe, C. L. van Oosten, C. W. M. Bastiaansen, J. Lub, and D. J. Broer, J. Mater. Chem. 15, 5043 (2005).

[4] C. L. van Oosten, K. D. Harris, C. W. M. Bastiaansen, and D. J. Broer, Eur. Phys. J. E 23, 329 (2007).

[5] Y. Yu, M. Nakano, and T. Ikeda, Nature (London) 425, 145 (2003).

[6] B. Serrano-Ramon, C. Kjellander, S. Zakerhamidi, C. W. M. Bastiaansen, and D. J. Broer, Proc. SPIE 6911, 6911091
(2008).

[7] K. Bhattacharya and R. D. James, Science 307, 53 (2005).

[8] E. Fried and S. Sellers, J. Chem. Phys. 124, 024908 (2006).

[9] N. Uchida, Phys. Rev. E 66, 040902(R) (2002).

[10] J. R. Frank and M. Kardar, Phys. Rev. E 77, 041705 (2008).

[11] J. Dervaux and M. Ben Amar, Phys. Rev. Lett. 101, 068101 (2008).

[12] M. M. Müller, M. Ben Amar, and J. Guven, Phys. Rev. Lett. 101, 156104 (2008).

[13] T. A. Witten, Rev. Mod. Phys. 79, 643 (2007).

[14] R. B. Meyer, Philos. Mag. 27, 405 (1973). 